\title{
Valor predictivo del tamizaje con tomografía computarizada en la detección de aneurismas aórticos en la población mexicana mayor de 55 años
}

Carlos A. Hinojosa, Javier E. Anaya-Ayala, Karla Bermúdez-Serrato, Paula Leal-Anaya, Hugo Laparra-Escareno y Adriana Torres-Machorro

Dirección de Cirugía, Sección de Angiología y Cirugía Vascular, Instituto Nacional de Ciencias Médicas y Nutrición Salvador Zubirán, Ciudad de México, México

\section{Resumen}

Objetivo: Determinar la prevalencia de aneurisma aórtico $(A A)$ mediante su búsqueda intencionada en estudios tomográficos en la población en riesgo. Método: Estudio observacional, transversal, que consistió en revisar de manera sistemática las tomografías computarizadas (TC) de tórax y abdomen realizadas por indicaciones diversas. Seleccionamos estudios de pacientes mayores de 55 años, del 1 de enero de 2014 al 31 de diciembre de 2016. Se llevó a cabo estadística descriptiva en la población estudiada y análisis de regresión lineal con el fin de determinar la relación del hallazgo de AA y la edad del paciente. Resultados: Fueron incluidos 4809 pacientes, de los cuales 2707 (56\%) eran de sexo femenino. El promedio de edad de la población estudiada fue de $69 \pm 9$ años. La prevalencia de AA fue del 5.63\% (271), con predominio en el sexo masculino (9.5 vs. 2.9\%). Del total, 138 (2.8\%) se detectaron en la aorta torácica, mientras que 133 (2.7\%) en la aorta abdominal. El modelo de regresión lineal demostró $Y=8.3+0.154$ y $r^{2}=0.03(p=0.001)$. Conclusiones: El tamizaje imagenológico con TC de $A A$ ha tenido un impacto favorable en nuestra institución y refuerza la necesidad de educar a los médicos radiólogos en la búsqueda intencionada de esta patología. Existe una correlación positiva entre la edad del paciente y el diámetro aórtico.

PALABRAS CLAVE: Aneurisma. Aorta. Tomografía computarizada. Tamizaje. Prevalencia.

\begin{abstract}
Objective: To determine the prevalence of aortic aneurysm $(A A)$ in tomographic studies in the population at risk. Methods: An observational, cross-sectional study that consisted in systematically reviewing computed tomographies (CT) of the chest and abdomen was carried out at our institution. These studies were performed for different clinical indications, we selected studies of patients older than 55 years from January 1, 2014 to December 31, 2016. Descriptive statistics was completed in the studied population and linear regression model was performed to determine the relationship of $A A$ findings and the patient's age. Results: A total of 4809 patients were included in this study, 2707 (56\%) were females. The mean age of the study population was $69 \pm 9$ years. The prevalence of aortic aneurysms was $5.63 \%(271)$ with a predominance in the male gender (9.5\% vs. $2.9 \%)$. Of the total, 138 (2.8\%) were located in the thoracic aorta and 133 (2.7\%) were in the abdominal aorta. Linear regression model demonstrated a $Y=8.3+0.154, r^{2}=0.03(p=0.001)$. Conclusions: Imaging screening with CT for aortic aneurysms has had a favorable impact in our institution and this reinforces the need to educate radiologists in the intentional search for this pathology. There is a positive correlation between the patient's age and aortic diameter.
\end{abstract}

KEY WORDS: Aneurysm. Aorta. Computed tomography. Prevalence.

\author{
Correspondencia: \\ Carlos A. Hinojosa \\ Vasco de Quiroga, 15 \\ Col. Belisario Domínguez, Sección XVI \\ C.P. 14080, Ciudad de México, México \\ E-mail: carlos.a.hinojosa@gmail.com
}

Fecha de recepción: 07-08-2017

Fecha de aceptación: 07-09-2017

DOI://dx.doi.org/10.24875/GMM.17000004
Gac Med Mex. 2017;153;Sup 2:S27-S33

Contents available at PubMed www.anmm.org.mx 


\section{Introducción}

Un aneurisma aórtico (AA) se define como una dilatación localizada de la aorta resultado del debilitamiento de sus paredes, pudiendo afectar uno o varios segmentos este vaso a lo largo de su trayectoria'. Se considera dilatación aneurismática aórtica cuando alcanza un diámetro igual o mayor a 1.5 veces el diámetro esperado en cada segmento, y de acuerdo con sus características anatómicas puede tener una forma fusiforme o sacular². Su localización más frecuente es la aorta abdominal, particularmente su porción infrarrenal $(90 \% \text { de los casos) })^{3,4}$ (Fig. 1). Los principales factores de riesgo que se han asociado a la patogénesis de AA abdominales incluyen el sexo masculino, el tabaquismo, los antecedentes heredofamiliares y padecimientos crónicos tales como hipertensión arterial sistémica, dislipidemias y enfermedad pulmonar obstructiva crónica ${ }^{5}$. Con respecto al sexo, la literatura reporta una prevalencia en los hombres entre 65 y 79 años de edad del $5-10 \%$, lo cual es cuatro a cinco veces mayor en que en las mujeres, en las que no se ha determinado con exactitud la prevalencia, pero se estima que es de alrededor del $1.3 \%{ }^{6}$.

Los AA son en su mayoría asintomáticos y en los países industrializados se diagnostican generalmente de manera incidental durante la realización de estudios de imagenología con tomografía computarizada (TC) o resonancia magnética (RM), o ultrasonido (US) en caso de la región abdominal, cuando estos estudios se indican por otra causa ${ }^{7,8}$. De presentar síntomas, el dolor torácico, abdominal o lumbar constituye la principal manifestación clínica en estos pacientes; en caso contrario, los aneurismas pueden diagnosticarse a partir de complicaciones tales como embolia distal, trombosis o compresión de estructuras adyacentes. La complicación más grave y que representa una de las emergencias vasculares más importantes es la rotura del aneurisma; se sabe que un retraso en su diagnóstico se asocia a un incremento en la mortalidad, que puede alcanzar hasta el 80\%9,10.

En los EE.UU., para los AA abdominales se ha estimado una mortalidad de 10,000 casos anuales ${ }^{11}$, y por ello el objetivo de los programas de tamizaje imagenológico realizados con US que se han puesto en marcha en ese y otros países industrializados desde la década pasada ha sido el de impactar en la tasa de rotura de aneurismas y, consecuentemente, en su mortalidad. En México todavía no existe un registro nacional de AA y aún se desconoce con certeza su prevalencia en nuestra población. En el Instituto Nacional de Ciencias Médicas y Nutrición Salvador Zubirán (INCMNSZ) se implementó, en el mes de octubre de 2015, un programa de escrutinio ultrasonográfico y un protocolo prospectivo en pacientes mayores de 65 años con factores de riesgo; para complementar el entendimiento sobre la prevalencia de esta enfermedad en nuestro medio, nosotros decidimos revisar de manera sistemática las TC y realizar una búsqueda intencionada de patología aórtica en

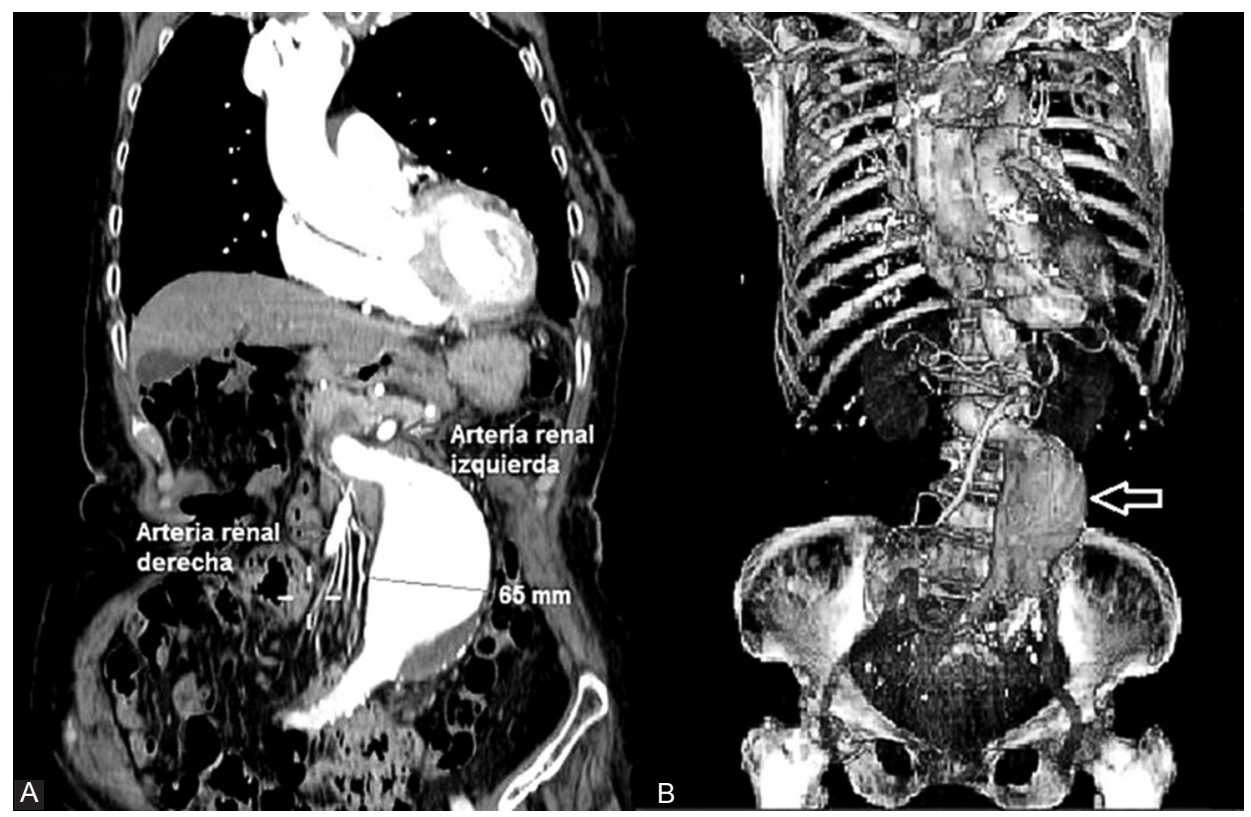

Figura 1. A: tomografía computarizada (vista coronal) en la que se observa un aneurisma de $65 \mathrm{~mm}$ de diámetro en la porción infrarrenal de la aorta abdominal. B: reconstrucción en tercera dimensión (3-D) de la aorta y el aneurisma (flecha). 
estudios realizados en un periodo de 2 años. El objetivo principal de este trabajo de investigación consistió en estudiar la prevalencia de AA y estimar el valor predictivo de la TC en pacientes con algún factor de riesgo.

\section{Métodos}

Nuestro trabajo consistió en un estudio transversal y observacional. Se utilizó el sistema de transmisión de imágenes Carestream Vue PACS (Siemens, Germany) para revisar de manera sistemática estudios de imagen colocando los siguientes filtros: modo TC, en pacientes de edad igual o mayor a 55 años con algún factor de riesgo, realizadas durante el periodo comprendido del 1 de enero de 2014 al 30 de diciembre de 2016. Cabe señalar que estos estudios se indicaron para detectar o descartar otra patología no aórtica. Se realizaron mediciones seriadas del diámetro anteroposterior de la aorta torácica ascendente y descendente, y de la aorta abdominal a nivel de la emergencia de la arteria mesentérica superior y de la arteria mesentérica inferior. Al encontrar una medición igual o mayor al $50 \%$ del diámetro esperado se realizó el diagnóstico de $A A$ y se reportaron la forma y la extensión del $A A$; además, se determinó la presencia de placas ateroescleróticas, trombo mural y calcificación de la aorta con su grado. Este estudio fue aprobado por el Comité de Ética de Investigación del INCMNSZ.

\section{Análisis estadístico}

Se realizaron estadística descriptiva y pruebas de $t$ de Student con el programa estadístico STATA versión 14.0 (Stata Corporation, College Station, USA) para encontrar diferencias en la población estudiada. Se realizó un análisis de regresión lineal para determinar la relación entre la edad del paciente y el diámetro aórtico.

\section{Resultados}

Un total de 4809 estudios tomográficos de pacientes del INCMNSZ fueron revisados tanto en la porción torácica como en la abdominal. De estos pacientes, $2707(56 \%)$ eran de sexo femenino y $2102(44 \%)$ de sexo masculino (Tabla 1). El promedio de edad de la población estudiada fue de $69 \pm 9$ años. La prevalencia encontrada de AA fue del $5.63 \%$ (271), con predominio en el sexo masculino (200 [9.5\%] vs. 71 [2.9\%]). Del total de estos, $133(2.7 \%)$ fueron abdominales y $35(26 \%)$ de ellos tenían un diámetro mayor de $5.5 \mathrm{~cm}$, que indica el inmediato tratamiento quirúrgico (Fig. 2). Se detectaron 138 (2.8\%) AA torácicos. La figura 3 ilustra la distribución, la localización y el número de los AA encontrados durante este estudio, así como los diferentes patrones anatómicos. La figura 4 representa el diagrama de cajas y bigotes, donde se observa la distribución por edad y sexo de los pacientes estudiados y el diámetro aórtico en milímetros. La figura 5 muestra el análisis de regresión lineal en la población estudiada; la edad se correlacionó con una dilatación. El modelo de regresión lineal mostró $Y=8.3+0.154$ y $^{2}=0.03(p=0.001)$.

\section{Discusión}

En México, la literatura respecto a la prevalencia de AA y el impacto en la salud pública es escasa o casi nula. En el año 2007, informes de la Secretaría de Salud reportaron un total de 4021 de AA que se presentaron con rotura entre 1984 y $2007^{12}$, lo cual probablemente representa una estadística por debajo de lo que podría ser la prevalencia real de esta enfermedad en la población mexicana, debido a que la rotura se manifiesta con muerte súbita y muchos de estos pacientes no llegan a recibir atención en un hospital

Tabla 1. Edad, sexo de los pacientes estudiados por tomografía computarizada y prevalencia de aneurismas aórticos

\begin{tabular}{lcc}
\hline Sexo & $\begin{array}{c}\text { Estudios } \\
\text { tomográficos }\end{array}$ & $\begin{array}{c}\text { Patología aórtica } \\
\text { aneurismática }\end{array}$ \\
\hline Masculino & $2102(44 \%)$ & $200(9.5 \%)$ \\
Femenino & $2707(56 \%)$ & $71(2.9 \%)$ \\
Total & 4809 & $271(5.63 \%)$ \\
\hline
\end{tabular}

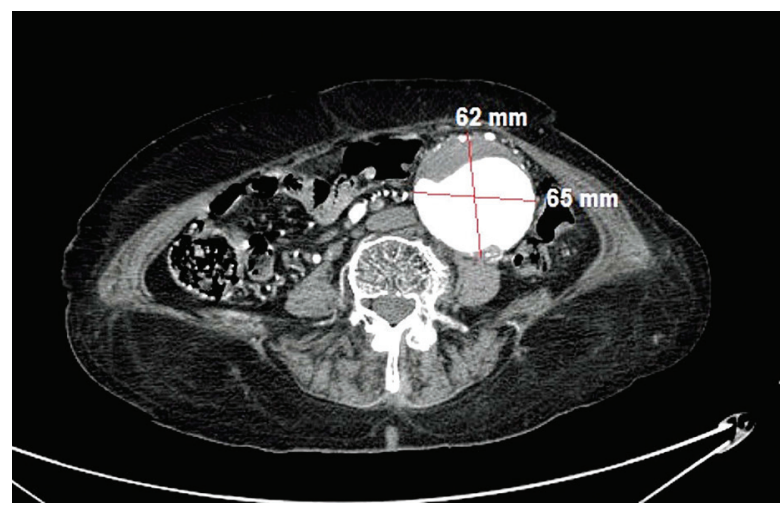

Figura 2. Tomografía computarizada que muestra la presencia de un aneurisma de $65 \mathrm{~mm}$ de diámetro por $62 \mathrm{~mm}$ en su diámetro antero posterior; dimensiones que cumplen indicación para tratamiento quirúrgico o endovascular. 


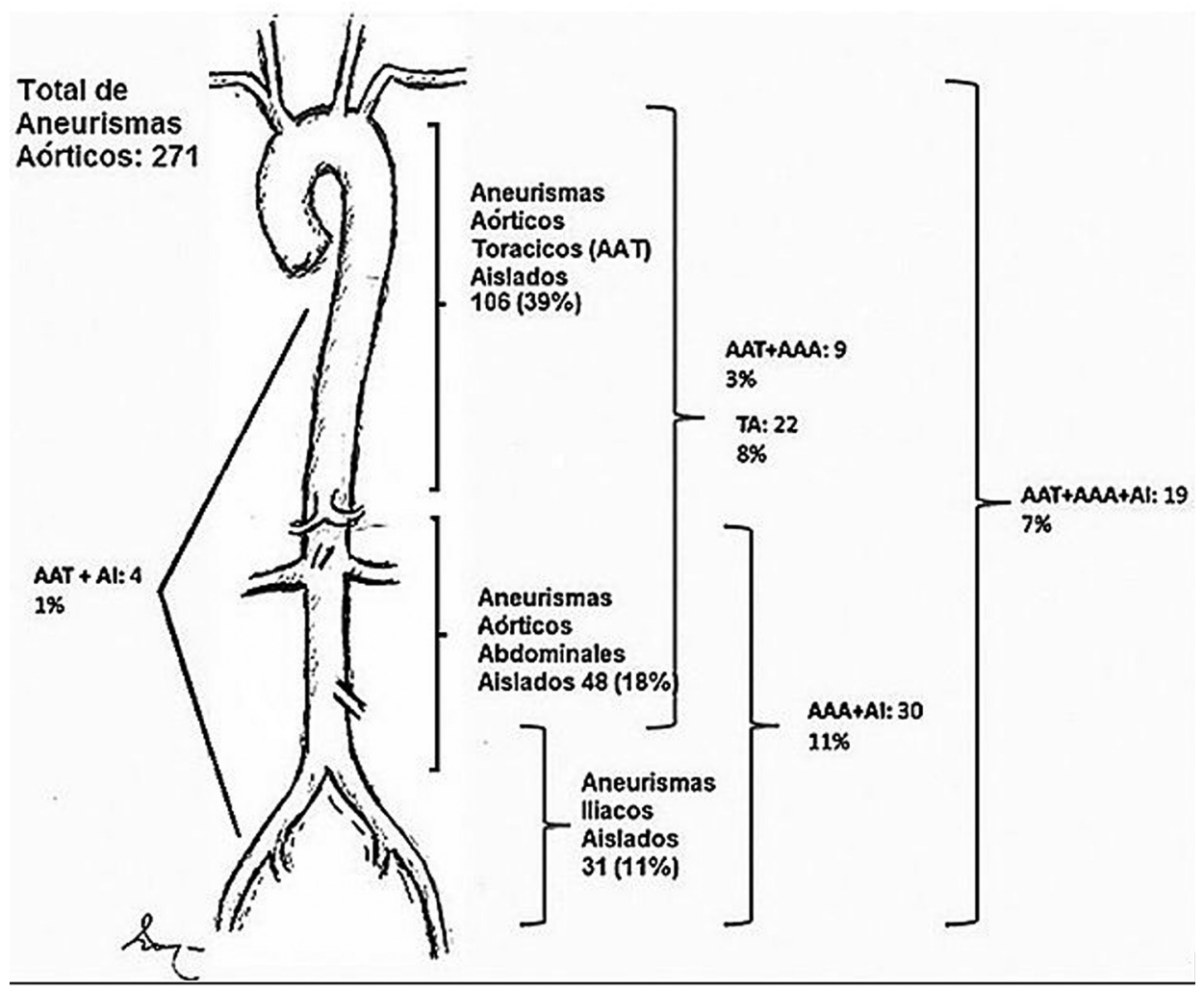

Figura 3. Esquema anatómico de la aorta y sus ramas que ilustra la distribución, el número, la localización y los patrones anatómicos la patología aneurismática aorta encontrada en este estudio. AAA: aneurismas aórticos abdominales; AAT: aneurismas aórticos torácicos; Al: aneurismas ilíacos.

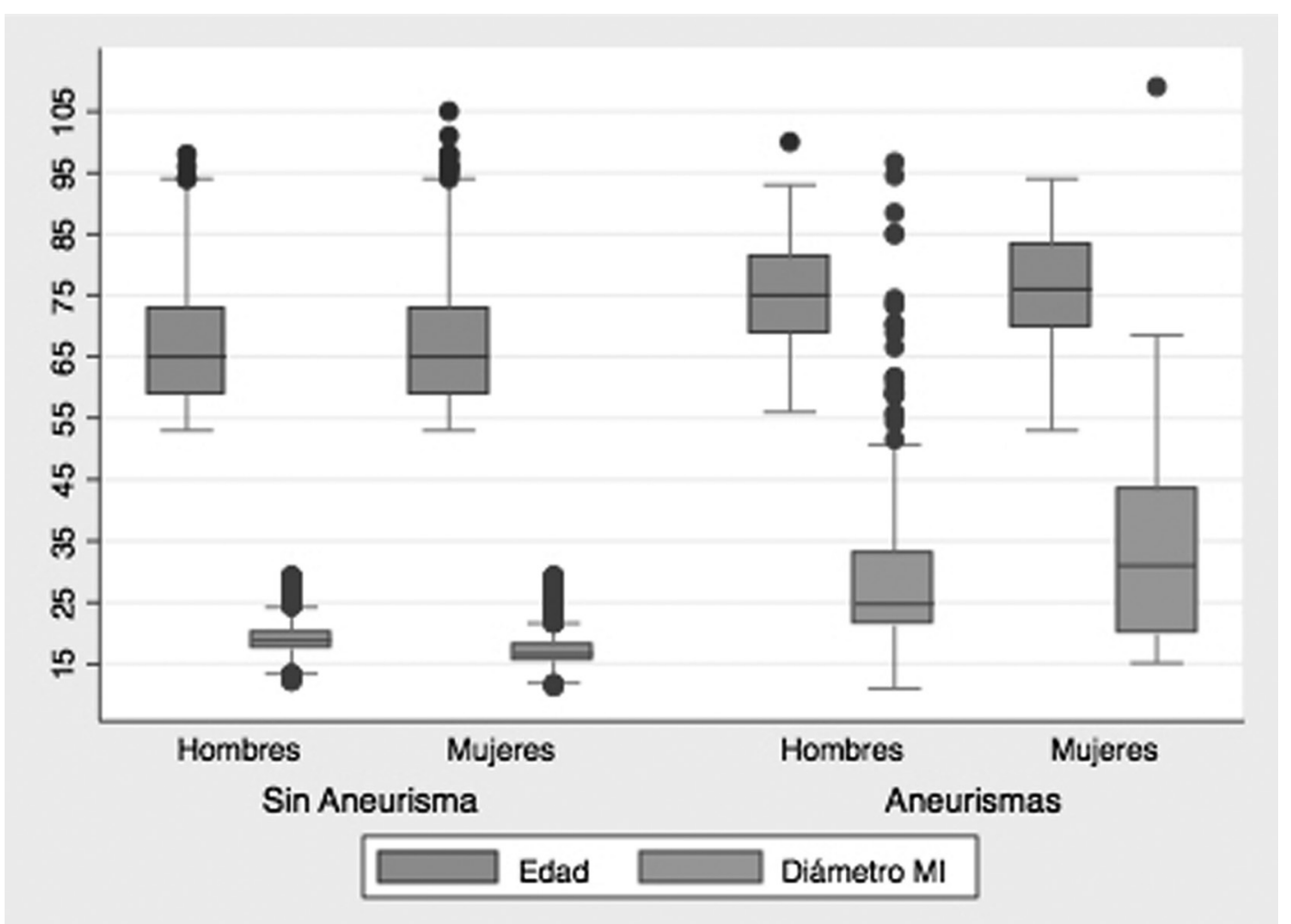

Figura 4. Diagrama de cajas y bigotes en el que se observan la distribución por edad y sexo de los pacientes estudiados y el diámetro aórtico en milímetros. 


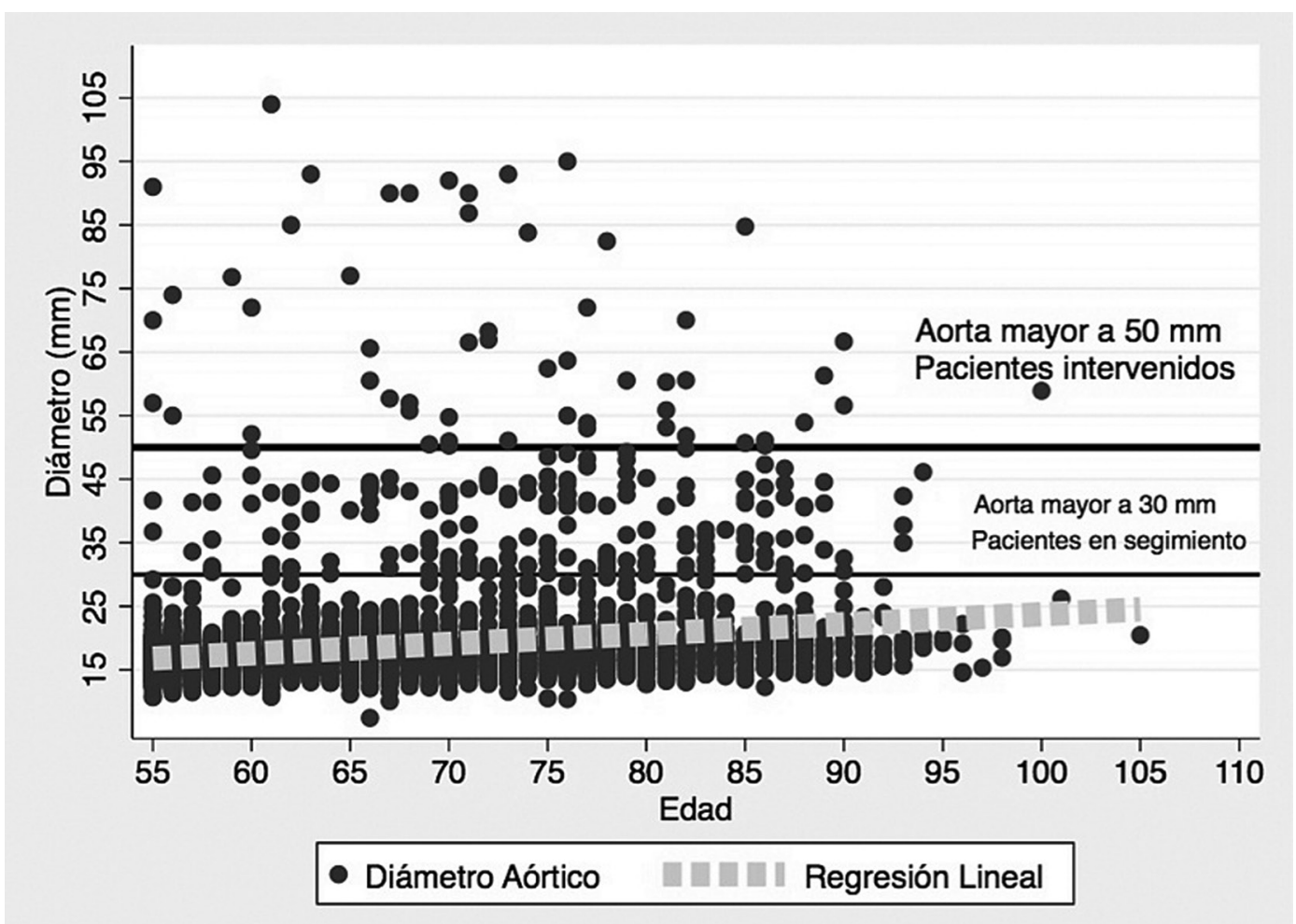

Figura 5. Gráfico de regresión lineal de la población estudiada. Se muestra la dispersión de aquellos pacientes con un diámetro menor de 30 $\mathrm{mm}$, mayor de $30 \mathrm{~mm}$ en seguimiento clínico, y mayor de $50 \mathrm{~mm}$ que han sido intervenidos (cirugía abierta y endovascular). Se observó una correlación positiva entre el diámetro aórtico y la edad. El modelo de regresión lineal mostró $Y=8.3+0.154$ y $r^{2}=0.03(p=0.001)$.

con los recursos humanos, científicos y tecnológicos para determinar el diagnóstico. La Guía de Práctica Clínica Mexicana sobre AA del Instituto Mexicano del Seguro Social (IMSS) menciona que en el $48 \%$ de los casos, los AA abdominales se descubren clínicamente, el $37 \%$ de manera incidental mediante estudios de imagen solicitados por otra causa y el $15 \%$ durante una intervención quirúrgica abdominal ${ }^{13}$. Por otro lado, en los EE.UU., donde en las últimas dos décadas el número de estudios de imagenología tales como la TC, la RM y la US han aumentado 20 veces en su número, y el 80 a $90 \%$ de los AA todavía son diagnosticados de manera incidental, se recomienda y realiza el tamizaje ultrasonográfico sistemático en pacientes con factores de riesgo ${ }^{14}$. La US abdominal es un estudio no invasivo de relativo bajo costo que tiene una sensibilidad del $95 \%$ y una especificidad cercana al $100 \%$ para la detección de $A A^{15}$. Los servicios de medicina preventivos en IOS EE.UU. recomiendan un estudio de tamizaje en hombres fumadores y con edad mayor de 65 años $^{16}$. Las guías clínicas de la Sociedad de Cirugía Vascular de los EE.UU. recomiendan la realización de estudios de US en hombres de 55 años o mayores, y en mujeres de 65 años o mayores, con historia de tabaquismo y antecedente familiar de aneurismas ${ }^{16}$. Estos programas han demostrado ser consistentemente efectivos en cuanto a costos para reducir la incidencia de rotura de AA y los costos asociados al tratamiento quirúrgico de urgencia por rotura, así como los días de estancia en la unidad de cuidados intensivos ${ }^{17}$. Se conoce que el riesgo de rotura es mayor cuando el diámetro aórtico es mayor de $5.5 \mathrm{~cm}$ en el sexo masculino y de $5 \mathrm{~cm}$ en las mujeres, y este riesgo aumenta de manera lineal con el incremento del diámetro ${ }^{18}$ (Fig. 6).

El estudio de escrutinio de AA abdominales de mayor escala que se ha realizado es el Multicentre Aneurysm Screening Study (MASS) en el Reino Unido, un ensayo clínico aleatorizado que incluyó 67.800 hombres de entre 65 y 74 años en cuatro centros hospitalarios. Dicho estudio tomó como punto de corte para indicación quirúrgica un diámetro de $5.5 \mathrm{~cm}$, mientras que a aquellos de $3-5.4 \mathrm{~cm}$ se les dio seguimiento, anual entre $3 \mathrm{y}$ $4.4 \mathrm{~cm}$, y trimestral entre 4.5 y $5.4 \mathrm{~cm}$. Se encontraron resultados similares al anterior, con una disminución del riesgo de mortalidad asociada a rotura del $42 \%{ }^{19}$.

En México, la guía práctica del IMSS hace énfasis en la necesidad de educar y concientizar a los expertos en radiología para la búsqueda intencionada de patología aórtica por parte de médicos radiólogos y 


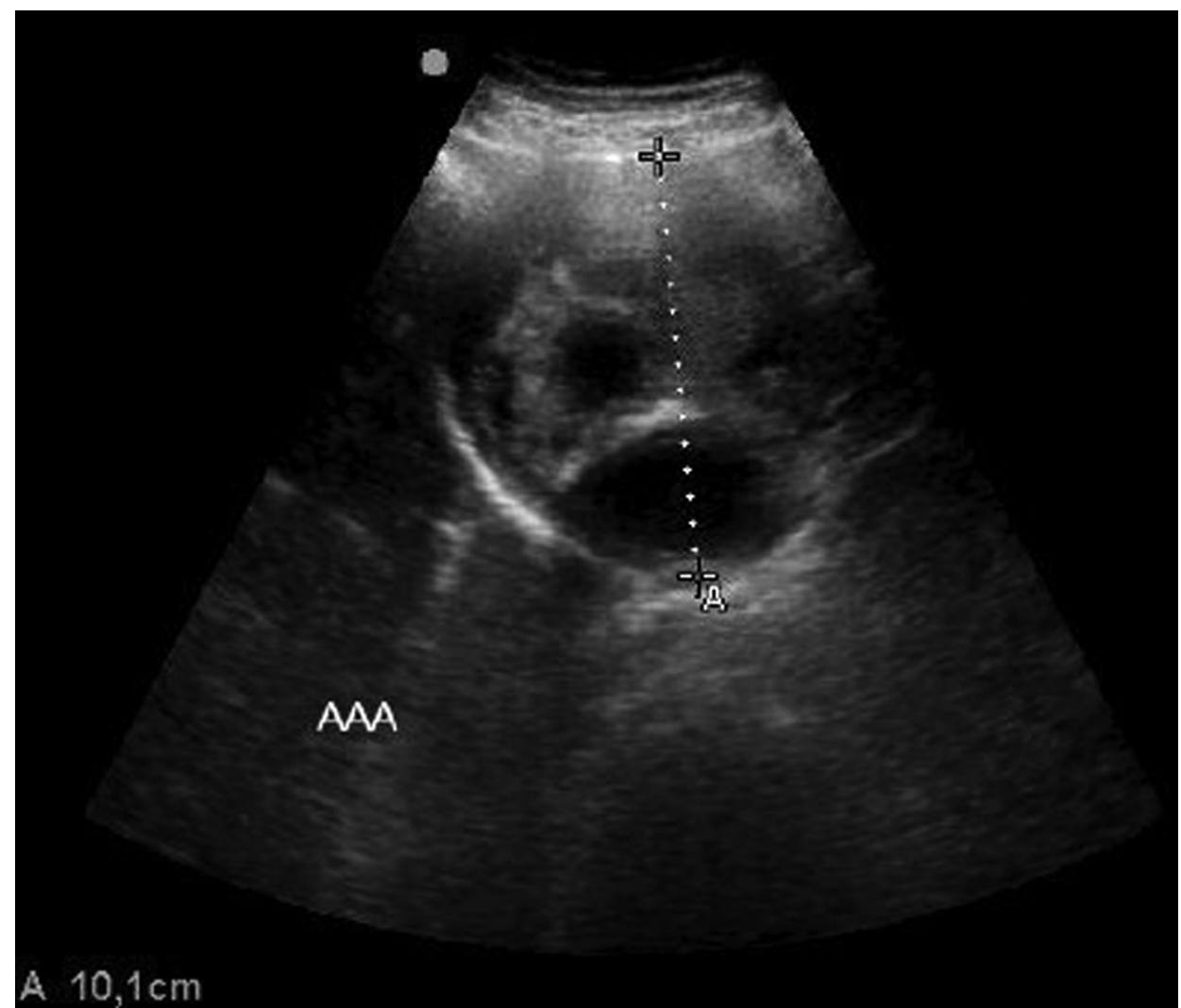

Figura 6. Estudio ultrasonográfico de la aorta abdominal que demuestra un diámetro anteroposterior del vaso de $10.1 \mathrm{~cm}$.

clínicos en todo paciente a quien se le realice US, TC o RM abdominal ${ }^{13}$. Identifica y confirma como población de riesgo a hombres mayores de 55 años con antecedente familiar de $\mathrm{AA}$ abdominal y en mayores de 65 aun cuando no se conozca. En las mujeres se recomienda en mayores de 65 años en caso de haber antecedentes de AA abdominal. En los pacientes con diámetro del aneurisma de 4-5.4 cm hay controversia sobre el seguimiento requerido ${ }^{13}$. En México, en el año 2015 llevó a cabo un estudio en 144 pacientes hospitalizados en el Hospital de Especialidades del IMSS, con una media de edad de 72.7 años, detectando una prevalencia del $6.9 \%$ en hombres $(95.1 \%$ de la muestra), con el cual la Institución se reitera en una detección sistemática mediante US Dúplex ${ }^{20}$.

La aorta abdominal es estructuralmente distinta de su porción torácica. Una de las principales diferencias es la cantidad de capas fibromusculares, las cuales proveen elasticidad y distribuyen el estrés ejercido en sus paredes. La capa media de la aorta torácica cuenta con alrededor de 60 unidades divididas en regiones vasculares y avasculares, mientras que la abdominal es completamente avascular y cuenta con alrededor de 30 unidades. Debido a las diferencias estructurales en los aneurismas torácicos y aórticos, generalmente no se presentan de manera concomitante, con solo un
$10-15 \%$ de probabilidad de que un individuo presente enfermedad aneurismática en ambas porciones de la aorta $^{21}$. Sin embargo, cabe señalar que en este estudio encontramos patología concomitante aislada en nueve pacientes y aneurismas con extensión toracoabdominal en 22 individuos.

El tamizaje imagenológico para la búsqueda intencionada y la detección de AA en el INCMNSZ ha confirmado que la prevalencia en la población mexicana no parece ser diferente a la reportada en la literatura en otros países y grupos étnicos. Reconocemos limitaciones metodológicas en nuestro estudio, incluyendo su naturaleza transversal y la posibilidad de un sesgo de selección, así como la falta de validación del modelo de regresión lineal de nuestro estudio. Sin embargo, se trata de una muestra importante de estudios imagenológicos en un gran número de pacientes en el tercer nivel de atención en nuestro país, donde todavía hace falta más información y concientización de esta patología entre médicos de otras especialidades.

\section{Conclusiones}

La búsqueda intencionada de patología aneurismática aórtica en nuestro Instituto parece impactar de manera favorable, encontrándose una prevalencia 
equivalente a la reportada en la literatura. Esto pone de manifiesto la necesidad de educar a la comunidad médica, así como al paciente con factores de riesgo, con respecto a dicha patología con el objetivo implementar de manera eficaz campañas de escrutinio que identifiquen a aquellos individuos con este padecimiento. Existe una correlación positiva entre la edad del paciente y el diámetro aórtico.

\section{Bibliografía}

1. Cosford $P$, Leng GC. Screening for abdominal aortic aneurysm. Cochrane Database Syst Rev. 2007;(2):CD002945.

2. Aboyans $V$, Bataille $V$, Bliscaux $P$, et al. Effectiveness of screening for abdominal aortic aneurysm during echocardiography. Am J Cardiol. 2014,1;114:1100-4.

3. Zankl AR, Schumacher $\mathrm{H}$, Krumsdrof $U$, et al. Pathology, natural history and treatment of abdominal aortic aneursysm. Clin Resp Cardiol. 2007; $96: 140-51$.

4. Singh K, Bønaa KH, Jacobsen BK, et al. Prevalence of and risk factors for abdominal aortic aneurysms in a population-based study: the Tromsø Study. Am J Epidemiol. 2001;154:236-44.

5. Lederle FA, Ultrasonographic screening for abdominal aortic aneurysms. Ann Intern Med. 2003;131:741-7.

6. Granel A, Sarandria R. Guía de práctica clínica. Aneurismas de aorta abdominal: ¿está justificado su rastreo sistemático? Evid Actual Pact Ambul. 2005;8:150-3.

7. Mussa FF. Screening for abdominal aortic aneurysm. J Vasc Surg. 2015;62:774-8.
8. Johansen K, Kohler TR, Nicholls SC, et al. Ruptured abdominal aortic aneurysm: the Harborview experience. J Vasc Surg. 1991;13:240-5; discussion 245-7.

9. Aslam A, Fisher CM, Thoo C, Neale ML, Thomas SD. Patients with Ruptured Abdominal Aortic Aneurysm Have Become Higher Risk. Ann Vasc Surg. 2017; 42:176-82.

10. Ernst CB. Abdominal aortic aneurysm. N Engl J Med. 1993;328:1167-72.

11. Murphy SL, Xu J, Kochanek KD. Deaths: final data for 2010. Natl Vital Stat Rep. 2010;61:1-117.

12. http://www.dgepi.salud.gob.mx/infoepi/index.htm. Consultada el 30 de enero de 2017.

13. Guía de práctica clínica: Aneurismas aórticos abdominales. Catálogo Maestro de Guías de Práctica Clínica; IMSS-412-10.

14. Powell JT, Greenhalgh RM. Clinical practice. Small abdominal aortic aneurysms. N Engl J Med. 2003;348:1895-901.

15. LeFevre ML; U.S. Preventive Services Task Force. Screening for abdominal aortic aneurysm: U.S. Preventive Services Task Force recommendation statement. Ann Intern Med. 2014;161:281-90.

16. Chaikof EL, Brewster DC, Dalman RL, et al. SVS practice guidelines for the care of patients with an abdominal aortic aneurysm: executive summary. J Vasc Surg. 2009;50:880-96.

17. Suckow B, Schanzer AS, Hoel AW, et al. A national survey of disease-specific knowledge in patients with an abdominal aortic aneurysm. $J$ Vasc Surg. 2016;63:1156-62.

18. Chun KC, Schmidt AS, Bains S, et al. Surveillance outcomes of small abdominal aortic aneurysms identified from a large screening program. J Vasc Surg. 2016;63:55-61.

19. Scott RA, Wilson NM, Ashton HA, et al. Influence of screening on the incidence of ruptured aortic aneurysm: 5-year results of a randomized controlled study. Br J Surg. 1995;82:1066-70.

20. Enríquez-Vega ME, Solorio-Rosete HF, Cossio-Zazueta A, et al. Detección oportuna de aneurismas de aorta abdominal en población de riesgo. Rev Med Inst Mex Seguro Soc. 2015;53(Supl 1):S100-3.

21. Kuivaniemi H, Ryer EJ, Elmore JR, et al. Understanding the pathogenesis of abdominal aortic aneurysms. Expert Rev Cardiovasc Ther. 2015;13:975-87. 